Acta Crystallographica Section D

\section{Biological Crystallography}

ISSN 1399-0047

\title{
Fingerprinting redox and ligand states in haemprotein crystal structures using resonance Raman spectroscopy
}

It is crucial to assign the correct redox and ligand states to crystal structures of proteins with an active redox centre to gain valid functional information and prevent the misinterpretation of structures. Single-crystal spectroscopies, particularly when applied in situ at macromolecular crystallography beamlines, allow spectroscopic investigations of redox and ligand states and the identification of reaction intermediates in protein crystals during the collection of structural data. Singlecrystal resonance Raman spectroscopy was carried out in combination with macromolecular crystallography on Swiss Light Source beamline X10SA using cytochrome $c^{\prime}$ from Alcaligenes xylosoxidans. This allowed the fingerprinting and validation of different redox and ligand states, identification of vibrational modes and identification of intermediates together with monitoring of radiation-induced changes. This combined approach provides a powerful tool to obtain complementary data and correctly assign the true oxidation and ligand state(s) in redox-protein crystals.

\section{Introduction}

It is essential that crystal structures of metalloproteins are assigned to the correct redox and ligand states in order to gain maximum functional information and to avoid, for example, the misinterpretation of catalytic mechanisms. In the case of haemproteins that bind small gaseous ligands, the ability to identify the nature and occupancy of a ligand where there may be limited differences in electron density is crucial. Recent developments in single-crystal micro-spectrophotometry, particularly when applied in situ on synchrotron macromolecular crystallography beamlines, allow systematic spectroscopic monitoring of redox and ligand states in the crystals that are used for structure determination. This enables identification of the initial state of the crystal and its comparison with solution data and also the ability to monitor changes induced by X-ray irradiation.

The application of UV-visible spectroscopy to protein single crystals has become well developed in recent years (Pearson \& Owen, 2009; Pearson et al., 2007; Owen et al., 2011; Orville et al., 2011; Ellis et al., 2008; Antonyuk \& Hough, 2011), while a number of beamlines, including those at the ESRF, NSLS (Stoner-Ma et al., 2011) and Swiss Light Source (SLS; Pompidor et al., 2013), also now have the capability to conduct Raman spectroscopy either in non-resonance (Carpentier et al., 2007; Katona et al., 2007; Bourgeois et al., 2009) or resonance (RR; Daughtry et al., 2012) modes. The preferred goal is to measure data from the X-ray-exposed portion of the same crystal from which the crystal structure is determined. Recent developments of the on-axis multi-mode
Received 21 December 2013 Accepted 20 February 2014

PDB references: $A x C Y T c p$, 4cda; 4cdv; 4cdy; 4cip; 4cjg; 4 cjo 
microspectrophotometer (Pompidor et al., 2013) at beamline X10SA of the SLS allow back-scattered resonance Raman (RR) data to be conveniently measured along the same axis as the X-ray beam (ensuring good alignment) with minimal or no impact on the efficiency of crystallographic data collection.

The gas-binding haemprotein cytochrome $c^{\prime}$ from the denitrifying bacterium Alcaligenes xylosoxidans (AxCYTcp) discriminates between nitric oxide (NO) and carbon monoxide $(\mathrm{CO})$ by binding them to opposite faces of haem while excluding the binding of molecular oxygen. In the absence of gaseous ligands, the haem Fe ion is five-coordinate (5c) with a proximal histidine ligand and a vacant distal coordination site. $\mathrm{CO}$ binds at the distal face to form a sixcoordinate (6c) adduct, while NO initially forms a $6 \mathrm{c}$ distal NO adduct and proceeds via a transient dinitrosyl intermediate to form the stable $5 \mathrm{c}$ proximal adduct, involving displacement of

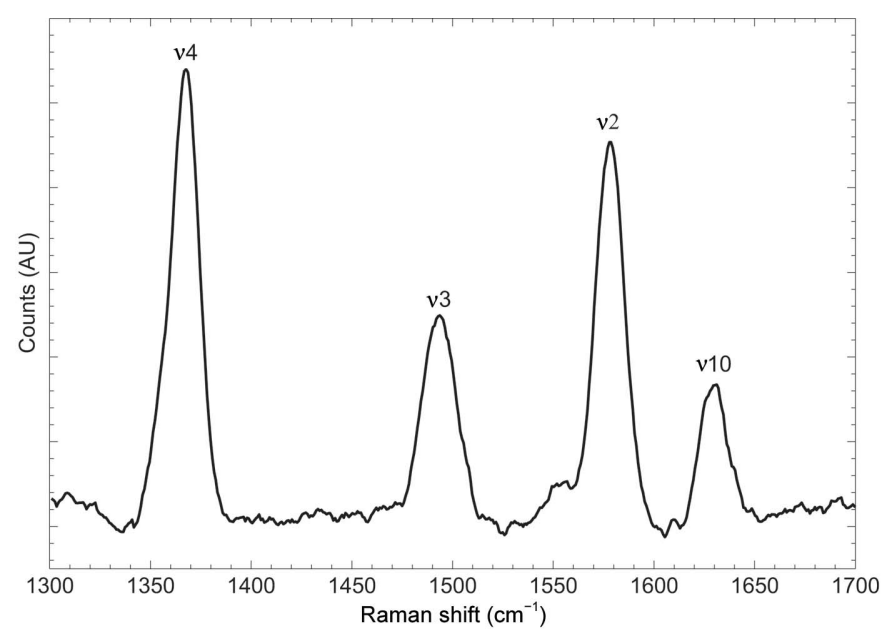

(a)

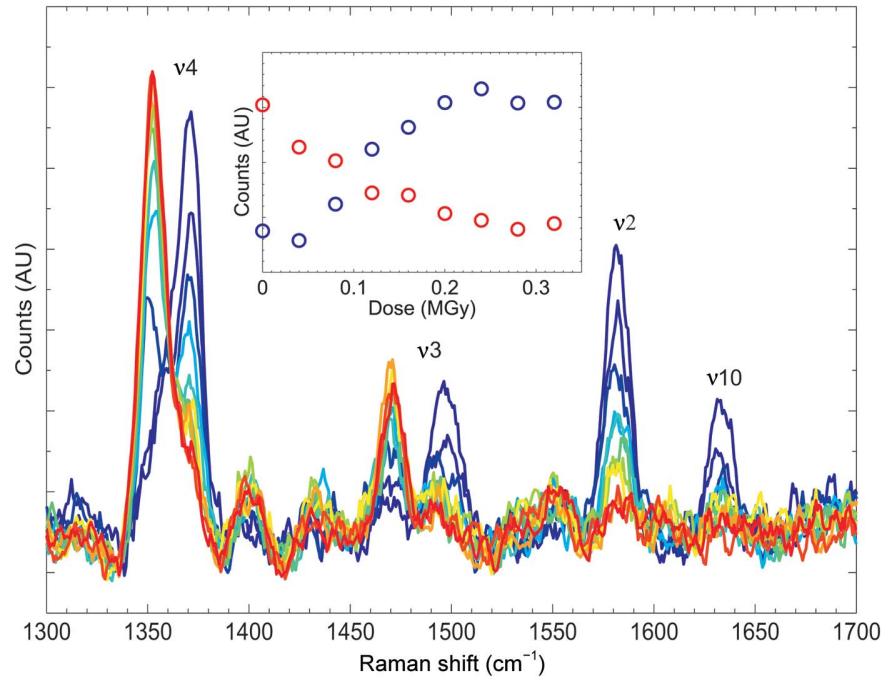

(b)

Figure 1 the proximal histidine (Andrew et al., 2002; Lawson et al., 2000). The 6c-to-5c conversion of NO ligation in cytochrome $c^{\prime}$ has recently been confirmed to be highly relevant to the mechanism of activation of soluble guanylate cyclase (Martin et al., 2012; Tsai et al., 2011), and proximal NO binding also occurs in the pro-apoptotic cytochrome $c$-cardiolipin complex (Silkstone et al., 2012). In neither of these cases are structural data available and $A x C Y$ Tcp provides a valuable structural model for NO binding.

Detailed solution RR studies have been performed on AxCYTcp (Andrew et al., 2001, 2002, 2005; Kruglik et al., 2007) and on a number of site-directed mutants (Antonyuk et al., 2011; Hough et al., 2011; Garton et al., 2012). Highresolution crystallographic analysis (Lawson et al., 2000; Antonyuk et al., 2011; Hough et al., 2011) of this protein and its mutants has provided detailed structural information, yet correlating crystallographic and solution spectroscopic data has remained a considerable challenge. The large irradiated surface area and very high resolution diffraction of $A x \mathrm{CYT} c \mathrm{p}$ crystals, together with the wealth of comparative solution data, make this protein an ideal test candidate for combined RR and crystallographic methods.

In this study, we present a comprehensive, correlated singlecrystal RR and structural study of $A x C Y T c p$ in its ferric, ferrous and gas ligand-bound forms as well as characterizing $\mathrm{X}$-ray-induced changes to these states. We demonstrate that redox and ligand states in crystal structures can be effectively spectroscopically fingerprinted in situ on the macromolecular crystallography beamline X10SA. This approach, if applied routinely to structural studies of haemproteins, has the potential to radically increase and complement the biological significance of the results gained from crystallographic experiments, such as redox-dependent biological mechanisms.

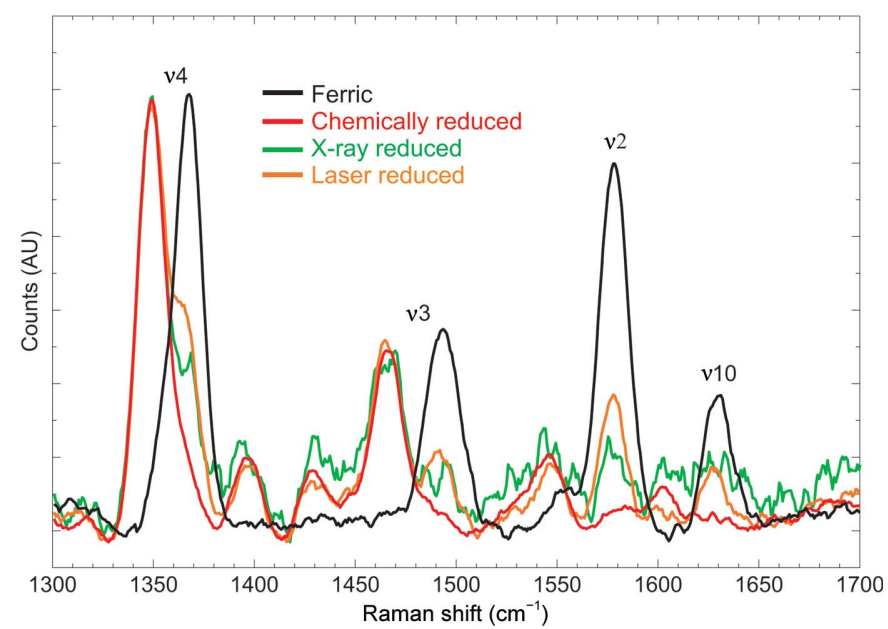

(c)

(a) RR spectra measured from a ferric $A x$ CYTcp crystal at $100 \mathrm{~K}$. The redox marker $v_{4}$ and the core size markers $v_{3}, v_{2}$ and $v_{10}$ are clearly defined. (b) $\mathrm{X}$-ray-reduction kinetic series of $20 \mathrm{~s}$ RR measurements. The first spectrum is shown in blue, with reduction essentially complete after an absorbed X-ray dose of $\sim 0.19 \mathrm{MGy}$. The inset shows the dose-dependent change in the bands at $1371 \mathrm{~cm}^{-1}$ (red) and $1352 \mathrm{~cm}^{-1}$ (blue). (c) RR spectra of ferric, chemically reduced, laser-reduced and X-ray-reduced crystals. Several spectra for each state were measured before X-ray exposure to ensure that no laser effects were apparent (except for the laser-reduced data) and only a single spectrum for each state is presented here. Data from a ferric crystal (black) were measured over $30 \mathrm{~s}$ at $\sim 2 \mathrm{~mW}$, data from an X-ray-reduced crystal (green) over $20 \mathrm{~s}$ at $\sim 0.88 \mathrm{~mW}$ and data from a chemically reduced crystal (red) over $30 \mathrm{~s}$ at $\sim 2 \mathrm{~mW}$. The laser-reduced spectrum shown (orange) was the final spectrum of a series of $20 \times 30 \mathrm{~s}$ at $\sim 4.8 \mathrm{~mW}$. Raman shifts measured from all three ferrous crystals are consistent within experimental error with solution data for the ferrous form of $A x C Y T c p$ (Table 1). 
Table 1

High-frequency Raman shifts $\left(\mathrm{cm}^{-1}\right)$ measured from crystals of $A x \mathrm{CYTcp}$ and comparative solution data at $100 \mathrm{~K}$.

n.o., not observed.

\begin{tabular}{lrllllll}
\hline & $T$ & \multicolumn{2}{l}{ Excitation } & & & \\
State & $(\mathrm{K})$ & $\lambda(\mathrm{nm})$ & $v_{4}$ & $v_{3}$ & $v_{2}$ & $v_{10}$ & Reference \\
\hline Ferric solution & 100 & 413.1 & 1370 & 1493 & 1581 & 1630 & C. R. Andrew (unpublished data) \\
Ferric crystal & 100 & 405.4 & 1369 & 1492 & 1577 & 1630 & This work \\
Chemically reduced crystal & 100 & 405.4 & 1350 & 1467 & 1582 & 1602 & This work \\
X-ray-reduced crystal & 100 & 405.4 & 1352 & 1466 & n.o & n.o & This work \\
Laser-reduced crystal & 100 & 405.4 & 1350 & 1467 & 1578 & n.o & This work \\
Ferrous solution & 90 & 413.1 & 1353 & 1471 & 1579 & 1607 Andrew et al. $(2002)$ \\
NO-bound solution & 90 & 413.1 & 1375 & 1504 & 1596 & 1638 Andrew et al. $(2002)$ \\
NO-bound crystal & 100 & 405.4 & 1378 & 1512 & 1597 & 1648 This work \\
CO-bound solution & 100 & 413.1 & 1370 & - & 1597 & 1634 Antonyuk et al. $(2011)$ \\
CO-bound crystal & 100 & 405.4 & 1369 & - & 1598 & - & This work \\
\hline
\end{tabular}

solid-state excitation laser (Omicron Laserage LDM405, 400, CWA) wavelength of $405.4 \mathrm{~nm}$. The Andor Shamrock 303 i spectrograph used a $24001 \mathrm{~mm}^{-1}$ grating, blazed at $300 \mathrm{~nm}$. Spectra were measured using an ANDOR Newton EMCCD DU-970N-UVB $(1600 \times 200$ pixels; Andor Technology PLC, Belfast, Northern Ireland) cooled to $193 \mathrm{~K}$ in full vertical binning mode. Raman shifts were calibrated using cyclohexane or 4acetamidophenol as a reference. Laser powers at the sample position were measured using a PMD100D hand-held power meter (Thorlabs Inc., New Jersey, USA). All spectra were processed and background-subtracted using the in-house $S L S-A P E$ analysis toolbox (Dworkowski et al., manuscript in preparation). Absorbed X-ray doses were calculated using the program RADDOSE-3D (Zeldin et al., 2013).

\section{Results}

\subsection{Measurement of resonance Raman data from AxCYTcp crystals and monitoring of $\mathrm{X}$-ray-reduction effects}

Resonance Raman (RR) data over the wavenumber range $600-2000 \mathrm{~cm}^{-1}$ were measured from native ferric $A x \mathrm{CYTcp}$ crystals using $405.4 \mathrm{~nm}$ laser excitation and on-axis geometry ${ }^{2}$ (Fig. 1 and Supplementary Fig. S1). The spectra showed good signal to noise, allowing a large number of Raman shifts to be detected characteristic of haem porphyrin groups. The spectrum measured from a ferric $A x C Y T c p$ crystal was consistent with equivalent solution data measured at $100 \mathrm{~K}$ (Table 1). Raman shifts in the haem porphyrin marker region are shown in Fig. 1(a) and Table 1. The values for the redox-state marker $v_{4}$ and the haem core size markers $v_{3}$ and $v_{10}$ lie within $1 \mathrm{~cm}^{-1}$ of those measured in solution, while the remaining core size marker $v_{2}$ is shifted by $4 \mathrm{~cm}^{-1}$ in the crystal data. These small differences are similar to the inherent resolution of the instrumentation $\left(\sim 3 \mathrm{~cm}^{-1}\right)$, but may also represent differences owing to the protein being in either a crystal lattice or in the solution state. Raman spectra taken from several different crystals of ferric $A x \mathrm{CYTcp}$ on separate visits to the beamline gave values for the porphyrin marker bands that were reproducible between $2-3 \mathrm{~cm}^{-1}$ (data not shown). Laser powers at the sample position ${ }^{3}$ were chosen below the threshold at which laser-induced photoreduction of the ferric haem is observed $(<2.4 \mathrm{~mW}$; Dworkowski et al., in preparation $\left.^{4}\right)$. Where appropriate, at least two consecutive

\footnotetext{
${ }^{2}$ Note that the laser light $(405.4 \mathrm{~nm})$ penetrates only a few micrometres into the crystal. An off-axis geometry without crystal reorientation would therefore not fully sample the X-ray-irradiated area.

${ }^{3}$ Laser powers given are those measured at the sample position unless otherwise noted.

${ }^{4}$ Note that for the laser spot size used, power densities are between $0.45 \mathrm{~mW} \mathrm{~mm}^{-2}$ (for $0.88 \mathrm{~mW}$ power) and $2.45 \mathrm{~mW} \mathrm{~mm}^{-2}(4.8 \mathrm{~mW}$ laser power).
}

\footnotetext{
${ }^{1}$ Supporting information has been deposited in the IUCr electronic archive (Reference: TZ5051).
} 
RR spectra in the absence of X-rays were taken for each measurement in order to exclude laser-induced effects on the crystals.

To investigate $\mathrm{X}$-ray reduction of the ferric haem in $A x \mathrm{CYTcp}$ crystals, a kinetic series of $50 \mathrm{RR}$ spectra, each of $20 \mathrm{~s}$ laser exposure, were measured using a laser power of $0.88 \mathrm{~mW}$. A 'blank' kinetic series measured first confirmed that no laser-induced spectral changes were evident before the $\mathrm{X}$-ray shutter was opened. Upon X-ray irradiation, the spectrum of ferric haem was rapidly interconverted to a spectrum with well resolved bands at 1352 and $1466 \mathrm{~cm}^{-1}$ (Fig. $1 b$, Table 1) corresponding to the ferrous protein. Complete conversion to the ferrous form was observed after a total absorbed X-ray dose of 0.19 MGy (0.024 MGy per spectrum; ${ }^{5}$ Fig. 1b).

\subsection{Measurement of a validated ferric haem crystal structure}

Given that rapid X-ray-induced reduction of the $A x C Y T c p$ haem iron(III) was observed, strategies were investigated to measure an 'intact' spectrum of the 'genuine' ferric form, and a multi-spot composite data-collection strategy was applied to large crystals (>1 mm maximum dimension) of ferric $A x C Y T c p$. This approach is analogous to that used to determine an intact state of an X-ray-sensitive ferryl intermediate in peroxidase (Meharenna et al., 2010). Five different positions along the crystal were chosen and marked using the bookmarking feature of the data-collection GUI. Partial data sets, each comprising $8^{\circ}$ crystal rotation, were measured, with the crystal translated to a new position between wedges. Rotation ranges at adjacent positions had a $2^{\circ}$ overlap in order to allow accurate scaling.

A single RR spectrum was measured from the crystal prior to X-ray exposure and at each bookmarked crystal position following X-ray data collection to ensure that no observable photoreduction had occurred (Supplementary Fig. S2). Each RR spectrum was collected at the $\omega$ angle in the centre of the rotation range used for the corresponding diffraction data wedge. A total of 32 diffraction images were obtained at each of the five positions at an X-ray wavelength of $0.8 \AA$, an X-ray flux of $2.97 \times 10^{10}$ photons s $^{-1}$ and a beam size of $50 \times 50 \mu \mathrm{m}$, which were processed and merged together to give a complete data set (Supplementary Table S1). With a highly attenuated X-ray beam the absorbed dose per data set was only $7.9 \mathrm{kGy}$. The resulting spectroscopically validated crystal structure for the ferric protein was determined to $1.3 \AA$ resolution. The structure shows the Arg124 side chain in a position parallel to the haem as previously proposed for the ferric state (Lawson et al., 2000). Structural parameters for the haem site of this and the other structures described herein are given in Table 2.

\subsection{Comparison of crystals reduced chemically and by X-ray irradiation}

Combined X-ray and RR data were measured from crystals reduced by different means: (i) chemical reduction using sodium ascorbate prior to crystal cooling, (ii) in situ reduction

\footnotetext{
${ }^{\mathbf{5}}$ The values quoted are the 'threshold average dose' calculated in RADDOSE-3D (Zeldin et al., 2013).
}

Table 2

Resolution and haem-ligand parameters for spectroscopically correlated AxCYTcp crystal structures.

\begin{tabular}{lllll}
\hline Structure & $\begin{array}{l}\text { Resolution } \\
(\AA)\end{array}$ & $\begin{array}{l}\mathrm{Fe}-\mathrm{His} 120 \mathrm{~N} \\
(\AA)\end{array}$ & $\begin{array}{l}\mathrm{Fe}-\mathrm{N} \\
(\mathrm{\AA})\end{array}$ & $\begin{array}{l}\mathrm{Fe}-\mathrm{N}-\mathrm{O} \\
\text { angle }\left(^{\circ}\right)\end{array}$ \\
\hline Ferric & 1.30 & 2.05 & - & - \\
Chemically reduced & 1.22 & 2.14 & - & - \\
X-ray-reduced $(100 \mathrm{~K})$ & 1.17 & 2.12 & - & - \\
X-ray-reduced $(160 \mathrm{~K})$ & 1.77 & 2.15 & - & - \\
X-ray-reduced $(180 \mathrm{~K})$ & 1.55 & 2.04 & - & - \\
5c NO complex & 1.26 & - & 1.84 & 42.1 \\
\hline
\end{tabular}

by exposure to $\mathrm{X}$-rays and (iii) reduction by exposure to a high-power $(\sim 4.8 \mathrm{~mW})$ excitation laser (600 s exposure) as described in Dworkowski et al. (in preparation). All three approaches yield similar spectra and indicate reduction of the initial ferric haem in the crystal to the ferrous state (Fig. 1c), with similar frequencies to those previously measured in solution (Table 1). Crystal structures were determined from chemically reduced crystals together with a crystal that had been reduced by X-rays, with confirmatory RR data measured to ensure that no further changes had occurred during crystallographic data collection. Owing to the limited penetration depth of the excitation laser of only a few micrometres (Adar et al., 2010), a crystal with a depth greater than this penetration depth cannot be fully laser-reduced. To avoid possible misinterpretation of a partially reduced state, we did not measure diffraction data from a laser-reduced sample.

The porphyrin RR frequencies of chemically reduced and $\mathrm{X}$-ray-reduced crystals are similar (Table 1). Interestingly, chemically and X-ray-reduced $A x$ CYTcp structures show large structural differences in the vicinity of the haem (Fig. 2), including the orientation of Arg124, previously proposed as a marker for sensing the Fe redox state. The ascorbate-reduced structure (Fig. 2b) agrees with previously reported ferrous $A x$ CYTcp structures in which the Arg124 guanadinium group is oriented perpendicular to the haem. By contrast, the X-rayreduced structure shows Arg124 parallel to the haem (Fig. 2c), in a similar orientation to the ferric state (Fig. 2a). We propose that the discrepancy between the chemically reduced and $\mathrm{X}$-ray-reduced structures is owing to the low $(100 \mathrm{~K})$ temperature of the crystal, such that conformational rearrangements in the haem pocket following photoreduction of haem $\mathrm{Fe}$ are restricted. To investigate this phenomenon further, the X-ray reduction experiment was repeated using fresh crystals at the elevated cryogenic temperatures of 160 and $180 \mathrm{~K}$. These temperatures are lower than those at which crystalline ice formation in the crystals occurs and thus goodquality diffraction data could still be obtained. As expected, the signal-to-noise of the RR data deteriorated rapidly as the temperature was increased, but reduction could still be monitored via the shift in the $v_{4}$ band to $1350 \mathrm{~cm}^{-1}$, although the other bands characteristic of the ferrous protein could not be detected above the noise level. ${ }^{6}$

\footnotetext{
${ }^{6}$ Interestingly, however, the chemically reduced crystal showed a small proportion (occupancy 0.2) of Arg124 in the 'ferric' orientation, suggesting that reduction was not complete prior to freezing of the crystal.
} 
The structure of $A x \mathrm{CYT}$ cp reduced by X-rays at $160 \mathrm{~K}$ was determined to a resolution of $1.77 \AA$, and in sharp contrast to the data set measured at $100 \mathrm{~K}$ it displayed two conformations

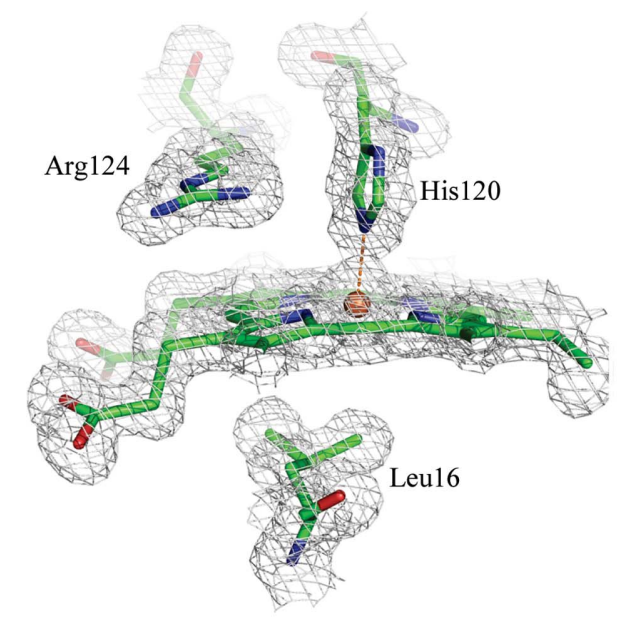

(a)

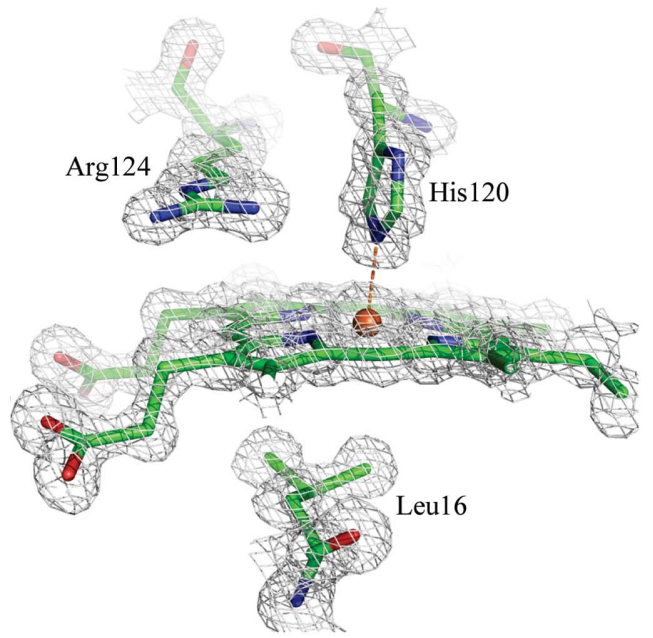

(c)

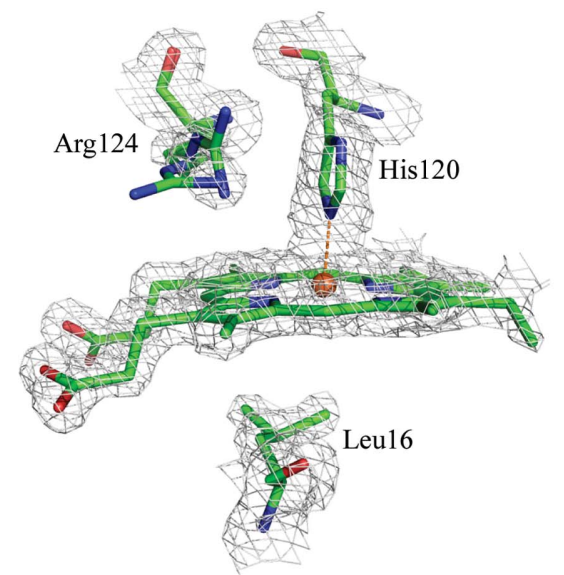

(e)

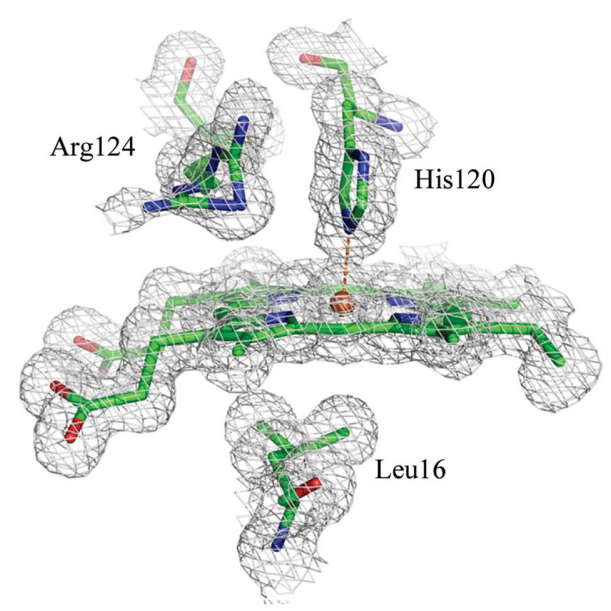

(b)

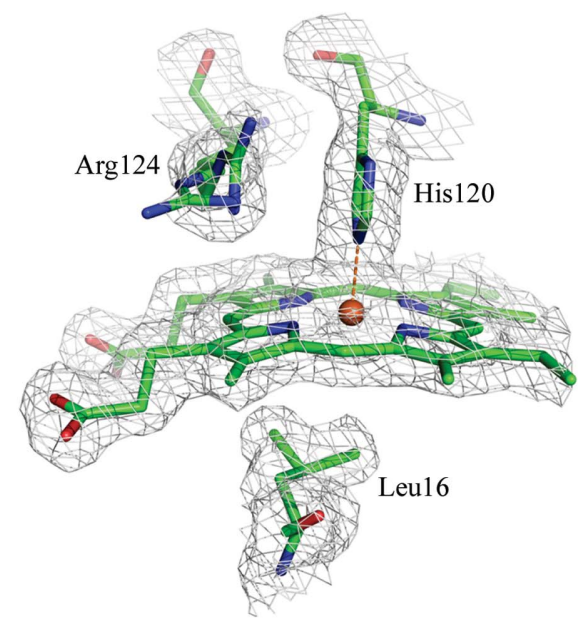

(d)

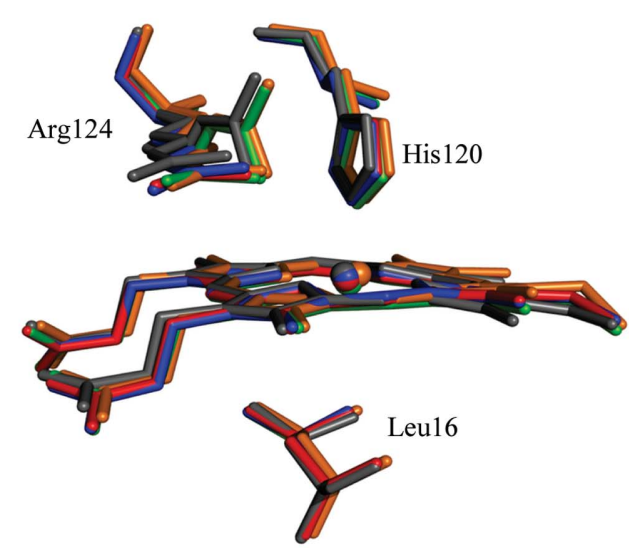

$(f)$
Figure 2

$2 F_{\mathrm{o}}-F_{\mathrm{c}}$ electron-density maps contoured at $1 \sigma$ showing the $A x \mathrm{CYT}$ cp crystal structures of $(a)$ ferric, $(b)$ chemically reduced and $(c) \mathrm{X}$-ray-reduced forms at $100 \mathrm{~K}$. Additional X-ray-reduced structures determined at different temperatures are shown at $160 \mathrm{~K}(d)$ and $180 \mathrm{~K}(e) .(f)$ Superposition of X-ray-reduced structures determined at $100 \mathrm{~K}$ (red), $160 \mathrm{~K}$ (grey) and $180 \mathrm{~K}$ (orange) with those of the chemically reduced (green) and ferric (blue) crystals. Note that in the chemically reduced, 160 and $180 \mathrm{~K}$ structures a minor occupancy conformation of Arg124 is present with density below the $1 \sigma$ level (see Supplementary Fig. S3). of Arg124, with the minor one (occupancy 0.3) similar to that present in the ferric structure and the major one (occupancy 0.7) corresponding to that in the chemically reduced crystal (Fig. 2b), indicating that conformational rearrangements supressed at $100 \mathrm{~K}$ were able to occur at higher temperatures. Additional data measured at $180 \mathrm{~K}$ showed a similar structure, again with a predominant 'chemically reduced ferrous' orientation of this side chain (occupancy 0.7) evident with a small component (occupancy 0.3) of the 'ferric' orientation. A superposition of the haem environments for the ferric, ascorbatereduced, X-ray-reduced (100 K), X-ray-reduced $(160 \mathrm{~K})$ and X-ray-reduced $(180 \mathrm{~K})$ structures is shown in Fig. $2(f)$. The Fe-His bond length was shortest $(2.05 \AA)$ in the composite ferric structure, becoming slightly longer in crystals reduced at $100 \mathrm{~K}(2.12 \AA)$ or reduced using ascorbate $(2.14 \AA)$. In the structures determined at 160 and $180 \mathrm{~K}$ the values were 2.15 and $2.04 \AA$, respectively, which are essentially the same given the lower resolution of these higher temperature structures.

The structures determined at 160 and $180 \mathrm{~K}$ exhibited a higher level of general radiation damage (beyond the haem pocket) than was the case at $100 \mathrm{~K}$. While no disulfide bonds are present in $A x$ CYTcp, decarboxylation and loss of side-chain electron density were very evident in the higher temperature structures. The unit cell underwent a small expansion at higher temperature, with an increase of $\sim 0.3 \AA$ in $a$ and $b$ and $\sim 1.0 \AA$ in $c$ between 100 and $160 \mathrm{~K}$, while no further change was apparent between 160 and $180 \mathrm{~K}$.

\subsection{Monitoring of gas ligand- bound states}

Data were measured from AxCYTcp crystals that had been chemically reduced with sodium 
ascorbate and exposed to either $\mathrm{NO}$ or $\mathrm{CO}$ prior to cooling to $100 \mathrm{~K}$ (Fig. 3). The CO-bound crystal exhibits porphyrin RR characteristic of a $6 \mathrm{c}$ low-spin species: $v_{4}\left(1369 \mathrm{~cm}^{-1}\right)$ and $v_{2}$ $\left(1598 \mathrm{~cm}^{-1}\right)$, with $v_{3}$ and $v_{10}$ modes not observed owing to low intensity. The RR spectrum for the NO-bound crystal is characteristic of a $5 \mathrm{c} \mathrm{NO}$ haem with porphyrin marker bands $v_{4}\left(1378 \mathrm{~cm}^{-1}\right), \quad v_{3}\left(1512 \mathrm{~cm}^{-1}\right), \quad v_{2}\left(1597 \mathrm{~cm}^{-1}\right)$ and $v_{10}$ $\left(1648 \mathrm{~cm}^{-1}\right)$ (Table 1$)$. The $v_{4}$ and $v_{2}$ frequencies of the $6 \mathrm{c} \mathrm{CO}$ and $5 \mathrm{c} \mathrm{NO}$ crystals at $100 \mathrm{~K}$ are similar to those of frozen solutions $(100 \mathrm{~K})$, although the $v_{3}$ and $v_{10}$ frequencies of the $5 \mathrm{c}$

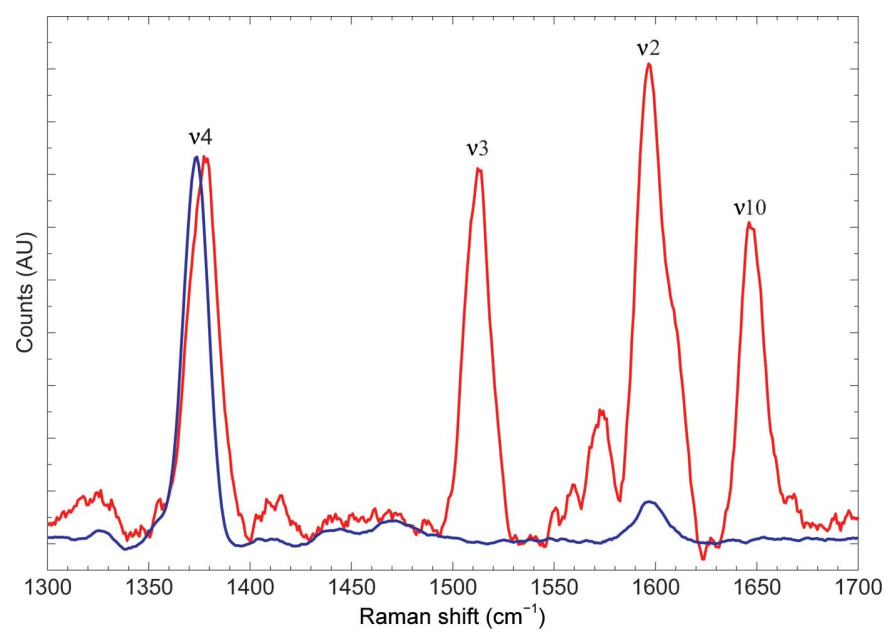

Figure 3

RR spectra measured from CO-bound (blue) and NO-bound (red) $A x$ CYTcp crystals. The sample laser power was $1.95 \mathrm{~mW}$ with $70 \mathrm{~s}$ exposures. The spectra show characteristic frequencies for $6 \mathrm{c}(\mathrm{CO})$ and $5 \mathrm{c}$ (NO) complexes, respectively, and a comparison with solution data is given in Table 1.

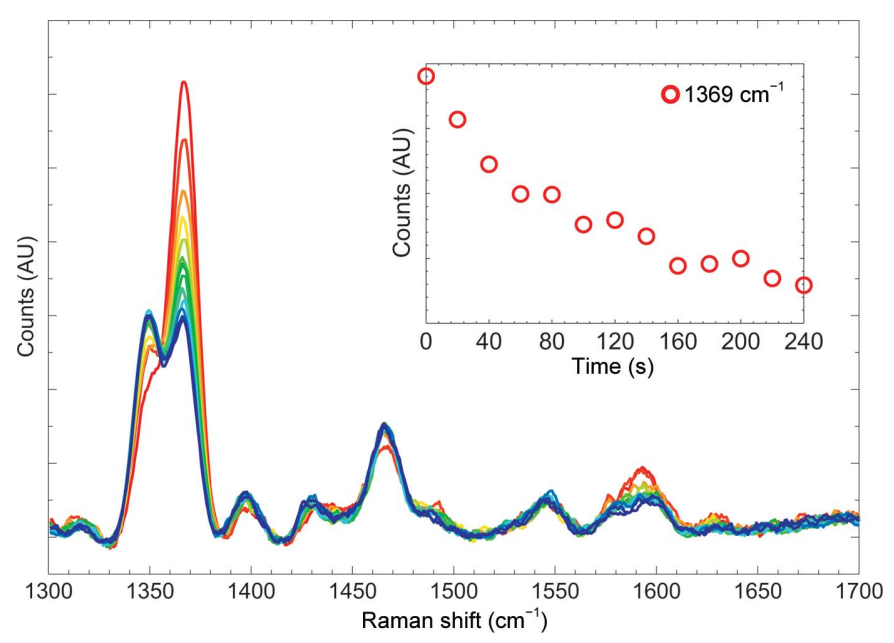

Figure 4

Consecutive resonance Raman spectra from a CO-bound crystal, each measured over $20 \mathrm{~s}$ of laser exposure at $\sim 5 \mathrm{~mW}$ power with no X-ray exposure for a total irradiation time of $240 \mathrm{~s}$. The starting (red) and final (blue) spectra are highlighted. Significant laser effects consistent with ligand dissociation were observed. Note that a degree of $\mathrm{CO}$ dissociation occurred even during measurement of the starting spectrum, as indicated by the shoulder in the $v_{4}$ band at $\sim 1350 \mathrm{~cm}^{-1}$ and the peak at $\sim 1465 \mathrm{~cm}^{-1}$. The inset shows the decay of the Raman band at $1369 \mathrm{~cm}^{-1}$ as a function of exposure time.
NO complex are $8-10 \mathrm{~cm}^{-1}$ higher in the crystalline state. This mirrors the trend for the ferric and ferrous states (Table 1), suggesting that the slight increases in the $v_{3}$ and $v_{10}$ frequencies are a specific feature of the crystalline state. No laserinduced dissociation of bound $\mathrm{NO}$ was observed in our experiments and the crystal structure (Supplementary Fig. S4) was essentially identical to that described previously (Hough et al., 2011). Our data are consistent with previous work where only X-ray dissociation of NO, not laser dissociation, was observed in haemoglobin crystals (Merlino et al., 2013). In contrast, the $A x \mathrm{CYT}$ - $-\mathrm{CO}$ complex was found to be highly susceptible to laser-induced ligand dissociation. At $5 \mathrm{~mW}$ laser power at the sample, progressive conversion from the ferrous $\mathrm{CO}$ spectrum to that of the ferrous $5 \mathrm{c}$ complex was observed in successive spectra (Fig. 4). This is consistent with, for example, flash-photolysis experiments, in which laser light at similar wavelengths can be used to dissociate $\mathrm{CO}$ from haem in order to investigate the kinetics of rebinding. We note here that dissociation will only be observed in the penetration depth of the laser into the crystal and thus this phenomenon, if not monitored and avoided, could cause a discrepancy between RR and crystallographic data measured on the same crystal.

\section{Discussion}

The combined RR and crystallographic data described here demonstrate that online RR spectroscopy is a powerful tool for fingerprinting both redox and ligand states in single crystals of haemproteins. RR spectroscopy allows information on vibrational states to be gained and, if performed on-axis, is particularly useful for large crystals, where UV-visible absorption microspectrophotometry is not feasible owing to the high optical density of the haemprotein crystals. High signal-to-noise RR spectra over the wavenumber range 600$2000 \mathrm{~cm}^{-1}$ were obtained from $A x$ CYTcp crystals at $100 \mathrm{~K}$ using low laser powers (up to $1.95 \mathrm{~mW}$ ) to avoid laser-induced reduction of haem or ligand dissociation. Characteristic spectra were measured for the ferric, ferrous, ferrous NO and ferrous $\mathrm{CO}$ states with clear resolution of the redox-marker band $v_{4}$ and the core size marker bands $v_{2}, v_{3}$ and $v_{10}$. The corresponding structures were comparable to those previously measured in crystallography-only experiments, but with an increased level of functional state reliability as a result of explicit validation of redox and ligand states.

RR data measured at $100 \mathrm{~K}$ from different redox and ligand states generally matched the corresponding solution data measured at the same temperature well. Some differences in frequencies occurred and this may be owing to the changes to vibrational modes imposed by the crystalline lattice. Nevertheless, ferric and ferrous states and $6 c$ (CO) and 5c (NO) complexes were clearly distinguished using RR data, allowing these biologically relevant changes to be monitored and followed by comparison to corresponding solution data. This approach can identify mixed populations in crystals, for example of ferric/ferrous oxidation state or incomplete ligand binding, although only in the region penetrated by the laser. 
RR spectra measured from crystals of ferric $A x \mathrm{CYTcp}$ before and after X-ray exposure showed significant changes owing to the conversion of ferric to ferrous protein, with reduction occurring following an X-ray dose of only 0.19 MGy. $\mathrm{X}$-ray photoreduction of haemprotein crystals has been well characterized (see, for example, Beitlich et al., 2007). In the case of $A x$ CYTcp, significant structural differences in the position of a proximal pocket arginine residue were observed between structures determined from a crystal reduced by $\mathrm{X}$-rays and from a crystal chemically reduced using sodium ascorbate prior to cooling, despite both having RR spectra consistent with the ferrous state. This apparent discrepancy was resolved by determination of the structures of X-rayreduced crystals at 160 and $180 \mathrm{~K}$, which contained electron density for both the 'ferric' and 'ferrous' arginine positions. Given these results, it is possible that the ferric crystals used for the determination of previous structures of $A x$ CYTcp may have undergone partial X-ray reduction of $\mathrm{Fe}$, but that given the lack of conformational changes observed between the ferric and X-ray-reduced structures at $100 \mathrm{~K}$ this was not apparent without complementary microspectrophotometric data.

The temperature at which protein crystals undergo the formation of crystalline ice and a catastrophic loss of crystalline order varies between crystals and is influenced by the size of the solvent channels (Weik, Kryger et al., 2001). In acetylcholinesterase (Weik, Kryger et al., 2001) this occurred at $>155 \mathrm{~K}$, whereas in $A x \mathrm{CYTcp}$ it did not occur at either 160 or $180 \mathrm{~K}$. The observation of partial occupancies of two distinct Arg124 conformers in our 160 and $180 \mathrm{~K} A x \mathrm{CYTcp}$ structures argues that there are two distinct, energetically favourable positions for the side chain and the flip from one to the other requires a combination of sufficiently high thermal energy and sufficiently low solvent viscosity. Weik, Kryger et al. (2001) proposed that a solvent glass transition occurs between 100 and $155 \mathrm{~K}$, with the higher temperature solvent displaying a greatly decreased viscosity that facilitates conformational changes in amino acids. Weik and coworkers have also demonstrated that product escape (Colletier et al., 2008) and side-chain flexibility (Weik, Ravelli et al., 2001) can be observed in structures of acetylcholinesterase measured at $155 \mathrm{~K}$ that were supressed at $100 \mathrm{~K}$.

Elevated temperatures above $150 \mathrm{~K}$ but below the temperature at which crystals are damaged by crystalline ice formation may facilitate the observation of relaxed or responsive structures (Weik \& Colletier, 2010). In the 160 and $180 \mathrm{~K}$ structures described here, in-crystal reduction by X-rays has been achieved while still maintaining crystalline order and diffracting power. The relatively lower temperature of $\sim 160$ $180 \mathrm{~K}$ prevents the 'destructive' level of radiation damage associated with room-temperature data collection, although more radiation damage is observed than at $100 \mathrm{~K}$.

High-quality characteristic vibrational frequencies of the porphyrin marker bands were obtained for both NO-bound and CO-bound states of $A x \mathrm{CYTcp}$, which were consistent with the obtained crystal structures and solution data. However, care must be taken when exposing CO-bound haemprotein crystals to laser light, as this can lead to dissociation of the $\mathrm{CO}$ ligand at high laser powers. Previous studies have shown that in myoglobin, photo-dissociated $\mathrm{CO}$ was able to migrate away from haem even at $20 \mathrm{~K}$ (Brunori et al., 2000).

Such effects are insidious as they will occur only in the part of the crystal penetrated by the laser beam and may therefore misrepresent the ligand status in the crystal as a whole. The data described here using a $405 \mathrm{~nm}$ solid-state excitation laser were limited to a lower wavenumber shift of $\sim 600 \mathrm{~cm}^{-1}$ owing to the notch filters used within the instrument. A number of important resonance Raman bands for $\mathrm{Fe}-\mathrm{CO}$ and $\mathrm{Fe}-\mathrm{NO}$ complexes lie at lower wavenumbers $\left(\sim 450\right.$ and $\sim 579 \mathrm{~cm}^{-1}$; Andrew et al., 2001), and future developments in the SLS instrumentation will aim to access this important spectral region.

In conclusion, high-resolution X-ray crystallography was effectively combined with resonance Raman spectroscopy to 'fingerprint' and validate different redox and ligand states in structures of $A x \mathrm{CYT}$. This combination of techniques provides a powerful tool to solve and correctly determine the true redox and ligand states in haemprotein crystals and would be valuable as a routine experimental procedure at advanced crystallographic beamlines. The combination of online spectroscopic techniques with X-ray crystallography is particularly important in micro-focused and nano-focused facilities, where the X-ray photon density will be much higher than the original facilities at the third-generation SR sources. The whole armoury of spectroscopic probes, e.g. UV-Vis, RR and XAS, have a significant role to play in providing functionally validated structures of redox proteins.

This work was carried out under SLS long-term beamtime awards 20090681 and 20111166 and was funded in part by EU FP7 BioStructX award 2370. DK holds a School Studentship at the University of Essex. We acknowledge the assistance of Dr Andrey Lebedev with restraint libraries in refinement and the JCSG for use of the Quality Control Check v.3.0 web server.

\section{References}

Adar, F., Lee, E., Mamedov, S. \& Whitley, A. (2010). Microsc. Microanal. 16, 360-361.

Andrew, C. R., George, S. J., Lawson, D. M. \& Eady, R. R. (2002). Biochemistry, 41, 2353-2360.

Andrew, C. R., Green, E. L., Lawson, D. M. \& Eady, R. R. (2001). Biochemistry, 40, 4115-4122.

Andrew, C. R., Kemper, L. J., Busche, T. L., Tiwari, A. M., Kecskes, M. C., Stafford, J. M., Croft, L. C., Lu, S., Moënne-Loccoz, P., Huston, W., Moir, J. W. \& Eady, R. R. (2005). Biochemistry, 44, 8664-8672.

Antonyuk, S. V. \& Hough, M. A. (2011). Biochim. Biophys. Acta, 1814, 778-784.

Antonyuk, S. V., Rustage, N., Petersen, C. A., Arnst, J. L., Heyes, D. J., Sharma, R., Berry, N. G., Scrutton, N. S., Eady, R. R., Andrew, C. R. \& Hasnain, S. S. (2011). Proc. Natl Acad. Sci. USA, 108, 1578015785 .

Beitlich, T., Kühnel, K., Schulze-Briese, C., Shoeman, R. L. \& Schlichting, I. (2007). J. Synchrotron Rad. 14, 11-23.

Bourgeois, D., Katona, G., de Rosny, E. \& Carpentier, P. (2009). Methods Mol. Biol. 544, 253-267. 
Brunori, M., Vallone, B., Cutruzzola, F., Travaglini-Allocatelli, C., Berendzen, J., Chu, K., Sweet, R. M. \& Schlichting, I. (2000). Proc. Natl Acad. Sci. USA, 97, 2058-2063.

Carpentier, P., Royant, A., Ohana, J. \& Bourgeois, D. (2007). J. Appl. Cryst. 40, 1113-1122.

Chen, V. B., Arendall, W. B., Headd, J. J., Keedy, D. A., Immormino, R. M., Kapral, G. J., Murray, L. W., Richardson, J. S. \& Richardson, D. C. (2010). Acta Cryst. D66, 12-21.

Colletier, J.-P., Bourgeois, D., Sanson, B., Fournier, D., Sussman, J. L., Silman, I. \& Weik, M. (2008). Proc. Natl Acad. Sci. USA, 105, 11742-11747.

Daughtry, K. D., Xiao, Y., Stoner-Ma, D., Cho, E., Orville, A. M., Liu, P. \& Allen, K. N. (2012). J. Am. Chem. Soc. 134, 2823-2834.

Ellis, M. J., Buffey, S. G., Hough, M. A. \& Hasnain, S. S. (2008). J. Synchrotron Rad. 15, 433-439.

Emsley, P., Lohkamp, B., Scott, W. G. \& Cowtan, K. (2010). Acta Cryst. D66, 486-501.

Evans, P. (2006). Acta Cryst. D62, 72-82.

Garton, E. M., Pixton, D. A., Petersen, C. A., Eady, R. R., Hasnain, S. S. \& Andrew, C. R. (2012). J. Am. Chem. Soc. 134, 14611463.

Henrich, B., Bergamaschi, A., Broennimann, C., Dinapoli, R., Eikenberry, E. F., Johnson, I., Kobas, M., Kraft, P., Mozzanica, A. \& Schmitt, B. (2009). Nucl. Instrum. Methods A, 607, 247-249.

Hough, M. A., Antonyuk, S. V., Barbieri, S., Rustage, N., McKay, A. L., Servid, A. E., Eady, R. R., Andrew, C. R. \& Hasnain, S. S. (2011). J. Mol. Biol. 405, 395-409.

Kabsch, W. (2010). Acta Cryst. D66, 125-132.

Katona, G., Carpentier, P., Nivière, V., Amara, P., Adam, V., Ohana, J., Tsanov, N. \& Bourgeois, D. (2007). Science, 316, 449-453.

Kruglik, S. G., Lambry, J. C., Cianetti, S., Martin, J. L., Eady, R. R., Andrew, C. R. \& Negrerie, M. (2007). J. Biol. Chem. 282, 50535062 .

Lawson, D. M., Stevenson, C. E., Andrew, C. R. \& Eady, R. R. (2000). EMBO J. 19, 5661-5671.

Martin, E., Berka, V., Sharina, I. \& Tsai, A. L. (2012). Biochemistry, 51, 2737-2746.
Meharenna, Y. T., Doukov, T., Li, H., Soltis, S. M. \& Poulos, T. L. (2010). Biochemistry, 49, 2984-2986.

Merlino, A., Fuchs, M. R., Pica, A., Balsamo, A., Dworkowski, F. S. N., Pompidor, G., Mazzarella, L. \& Vergara, A. (2013). Acta Cryst. D69, 137-140.

Murshudov, G. N., Skubák, P., Lebedev, A. A., Pannu, N. S., Steiner, R. A., Nicholls, R. A., Winn, M. D., Long, F. \& Vagin, A. A. (2011). Acta Cryst. D67, 355-367.

Orville, A. M., Buono, R., Cowan, M., Héroux, A., Shea-McCarthy, G., Schneider, D. K., Skinner, J. M., Skinner, M. J., Stoner-Ma, D. \& Sweet, R. M. (2011). J. Synchrotron Rad. 18, 358-366.

Owen, R. L., Yorke, B. A., Gowdy, J. A. \& Pearson, A. R. (2011). J. Synchrotron Rad. 18, 367-373.

Pearson, A. R. \& Owen, R. L. (2009). Biochem. Soc. Trans. 37, 378-381.

Pearson, A. R., Pahl, R., Kovaleva, E. G., Davidson, V. L. \& Wilmot, C. M. (2007). J. Synchrotron Rad. 14, 92-98.

Pompidor, G., Dworkowski, F. S. N., Thominet, V., Schulze-Briese, C. \& Fuchs, M. R. (2013). J. Synchrotron Rad. 20, 765-776.

Saavedra, J. E., Southan, G. J., Davies, K. M., Lundell, A., Markou, C., Hanson, S. R., Adrie, C., Hurford, W. E., Zapol, W. M. \& Keefer, L. K. (1996). J. Med. Chem. 39, 4361-4365.

Silkstone, G., Kapetanaki, S. M., Husu, I., Vos, M. H. \& Wilson, M. T. (2012). Biochemistry, 51, 6760-6766.

Stoner-Ma, D., Skinner, J. M., Schneider, D. K., Cowan, M., Sweet, R. M. \& Orville, A. M. (2011). J. Synchrotron Rad. 18, 37-40.

Tsai, A. L., Berka, V., Sharina, I. \& Martin, E. (2011). J. Biol. Chem. 286, 43182-43192.

Weik, M. \& Colletier, J.-P. (2010). Acta Cryst. D66, 437-446.

Weik, M., Kryger, G., Schreurs, A. M. M., Bouma, B., Silman, I., Sussman, J. L., Gros, P. \& Kroon, J. (2001). Acta Cryst. D57, 566-573.

Weik, M., Ravelli, R. B. G., Silman, I., Sussman, J. L., Gros, P. \& Kroon, J. (2001). Protein Sci. 10, 1953-1961.

Winn, M. D. et al. (2011). Acta Cryst. D67, 235-242.

Zeldin, O. B., Gerstel, M. \& Garman, E. F. (2013). J. Synchrotron Rad. 20, 49-57. 